\title{
GASTRECTOMÍA EN MANGA: ESTUDIO POR IMÁGENES DE SUS COMPLICACIONES*
}

\author{
Drs. Daniel Pérez C. ${ }^{1}$, Giancarlo Schiappacasse F. ${ }^{1}$, Francisco Zúñiga F. ${ }^{1}$, \\ Pablo Soffia S. ${ }^{1}$, Claudio Cortés A. ${ }^{1,2}$ \\ Servicio de Radiología, Facultad de Medicina Clínica Alemana-Universidad del Desarrollo. \\ 2 Servicio de Radiología Hospital Clínico de la Universidad de Chile. \\ Santiago, Chile.
}

\begin{abstract}
Laparoscopic sleeve gastrectomy: imaging study of its complications
\end{abstract}

The incidence of obesity in Chilean adult population reaches up to $25.1 \%$, according to statistics from 2013. Bariatric surgery is an alternative that has proven weight loss and resolution of comorbidities significant long term. For this reason are routine procedures in different health institutions. However, its massification and safety are not free of complications, which can be detected and treated early with the help of radiological images. The aim of this paper is to describe the most common postoperative complications of the sleeve gastrectomy and its diagnosis using different imaging techniques, mainly fluoroscopic study contrasted of the the esophagus-stomach-duodenum and contrasted enhanced computed tomography $(\mathrm{CT})$ of the abdomen and pelvis. Bariatric surgeries are now routine procedures for treatment of morbid obesity. Knowledge of early and late complications and diagnosis of these using different imaging techniques allow adequate treatment, control and decreased postoperative morbidity and mortality.

Key words: Morbid obesity, bariatric surgery, laparoscopic sleeve gastrectomy, complications.

\section{Resumen}

La incidencia de obesidad en la población adulta chilena alcanza hasta un $25,1 \%$, de acuerdo a cifras del año 2013. Las cirugías bariátricas son una alternativa que ha demostrado una baja de peso y resolución de comorbilidades significativas a largo plazo. Por este motivo son procedimientos rutinarios en las diferentes instituciones de salud. No obstante su masificación y seguridad, no están exentas de complicaciones, las que se pueden detectar y tratar precozmente con la ayuda de imágenes radiológicas. El objetivo de este trabajo es describir las complicaciones postquirúrgicas más frecuentes de la gastrectomía en manga y el diagnóstico mediante diferentes técnicas de imagen, principalmente el estudio fluoroscópico contrastado del esófagoestómago-duodeno y tomografía computada (TC) contrastada de abdomen y pelvis. El conocimiento de las complicaciones tempranas y tardías, así como el diagnóstico de éstas mediante las diferentes técnicas de imagen permitirá un adecuado tratamiento, control post operatorio y disminución de la morbimortalidad.

Palabras clave: Obesidad mórbida, cirugías bariátricas, gastrectomía en manga, complicaciones.

*Recibido el 23 de diciembre de 2014 y aceptado para publicación el 5 de marzo de 2015.

Los autores no refieren conflictos de interés.

Correspondencia: Dr. Daniel Pérez C. jdperezc@gmail.com 


\section{Introducción}

La incidencia de obesidad en población adulta chilena alcanza hasta un $25,1 \%$ y la obesidad mórbida un $1,3 \%$ aproximadamente ${ }^{1-3}$. Algunos autores postulan que la terapia basada en dieta y fármacos ha fracasado como tratamiento efectivo y duradero contra la obesidad ${ }^{3}$. Las cirugías bariátricas han demostrado que pueden lograr una baja de peso sustancial, además de una resolución de las comorbilidades asociadas a corto y largo plazo $^{3}$. Por este motivo las cirugías bariátricas son procedimientos rutinarios en muchas instituciones de salud ${ }^{1-3}$.

El objetivo de este estudio es describir las complicaciones postquirúrgicas más frecuentes de la gastrectomía en manga. Para ello fueron seleccionados casos representativos de Clínica Alemana de Santiago, del Hospital Clínico de la Universidad de Chile y del Hospital Padre Hurtado.

Las cirugías bariátricas se dividen en tres grupos según el mecanismo mediante el cual logran la reducción de la ingesta calórica: restrictivas, malabsortivas y combinadas. En las primeras, el tracto gastrointestinal es sustancialmente reducido de volumen para disminuir la ingesta y producir saciedad precoz. En el segundo tipo el tracto gastrointestinal es alterado quirúrgicamente para reducir la absorción de nutrientes. En el tercer tipo se combinan ambos procedimientos ${ }^{2,4}$.

La gastrectomía en manga laparoscópica fue inicialmente propuesta como un procedimiento previo al by-pass gástrico en pacientes obesos mórbidos, con índice de masa corporal sobre 50, y con alto riesgo operatorio. Es una técnica puramente restrictiva. Consiste en realizar un tubo gástrico de aproximadamente $150 \mathrm{cc}$ a partir de la curvatura menor del estómago, utilizando suturas tipo engrapadora o stapler con el fin de disminuir el volumen del estómago. En los últimos años se ha convertido en un procedimiento quirúrgico definitivo para el tratamiento de la obesidad debido a sus buenos resultados ${ }^{4,5}$.

Dentro de las complicaciones más frecuentes se encuentran las filtraciones, hemorragia, formación de abscesos, fístulas, estenosis y obstrucción e hiperacidez $z^{2,5,6}$.

\section{Filtraciones}

Corresponden al paso de contenido gástrico fuera del lumen. Los síntomas más frecuentes son taquicardia, leucocitosis, aumento de la proteína $\mathrm{C}$ reactiva (PCR) y fiebre 5 .

Se deben buscar en la línea de sutura gástrica y son más frecuentes en su margen superior $(1,5 \%$ versus $0,5 \%$ de filtración baja $)^{6}$. Si ocurren dentro de las primeras $48 \mathrm{~h}$, suelen deberse a fallas en la técnica quirúrgica. Aquellas que se presentan posteriormente pueden ser de origen isquémico.

El método de estudio más adecuado en el período post-operatorio temprano es la fluoroscopia con medio de contraste oral hidrosoluble (tránsito esofagogastroduodenal). Si la sospecha es alta y el estudio con contraste oral es negativo, se recomienda realizar una TC con uso de contraste oral, que puede demostrar colecciones y filtraciones ${ }^{2,6,7}$.

Dentro de los signos tomográficos que se pueden encontrar ante la presencia de una filtración debe destacarse como signo directo la presencia de salida de contraste hidrosoluble administrado por vía oral a la cavidad peritoneal, en relación al lecho quirúrgico y en particular a la línea de suturas. Como signos indirectos se encuentran las burbujas de gas y las colecciones líquidas ubicadas adyacentes al lecho quirúrgico. Cabe destacar que las burbujas de gas pueden corresponder a un hallazgo habitual dentro de los primeros 5 a 7 días, siendo inusual encontrarlas con posterioridad a este período (Figuras 1 y 2).

Algunos estudios han demostrado que la instalación de un stent endoscópico auto-expandible en la zona anatómica de filtración es efectivo para el control de éstas ${ }^{8}$ (Figura 3).

\section{Hemorragia}

El riesgo de sangrado de la gastrectomía en manga varía entre 1 a $1,6 \% \%^{5}$. El sitio más frecuente es la línea de sutura, pero también puede originarse en vasos u órganos cercanos. Es importante una adecuada valoración pre-operatoria con el fin de identificar condiciones médicas, anatómicas y fármacos que aumenten el riesgo de sangrado durante la cirugía ${ }^{5,6}$.

En el período post-operatorio, si el paciente es sintomático o hay caídas en el hematocrito, se sugiere estudio mediante TC con contraste intravenoso, la que puede identificar el sitio y estimar la cuantía del sangrado ${ }^{2,5,6}$.

$\mathrm{La}$ endoscopia digestiva alta puede tener un rol diagnóstico o terapéutico en las hemorragias intraluminales ${ }^{5,6}$.

El tratamiento de las hemorragias y hematomas variará según el sitio y cuantía de éstos. Es posible un tratamiento expectante en casos intraluminales. En casos severos, la re-exploración quirúrgica puede ser la única forma de detener sangrados incoercibles $^{2,5}$ (Figura 4).

\section{Colecciones y abscesos}

Se originan a partir de filtraciones. El método de estudio es la TC con contraste intravenoso, pudiendo mostrar colecciones líquidas definidas por una pared que realza con el medio de contraste ${ }^{2,5,6}$. 


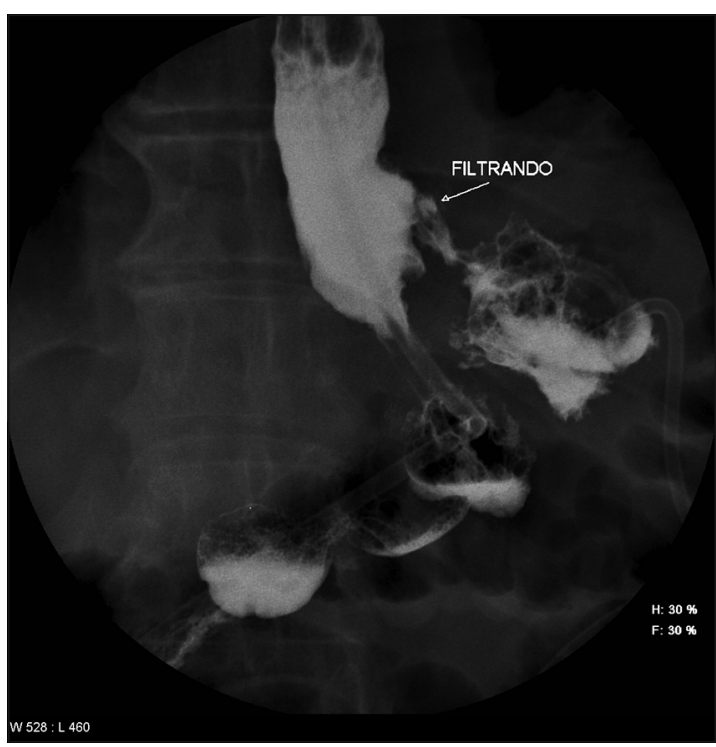

Figura 1. Tránsito esofagogastroduodenal con contraste hidrosoluble Control post-gastrectomía en manga reciente. Salida del medio de contraste deglutido a la cavidad peritoneal, a través de la región más cefálica de la línea de sutura.

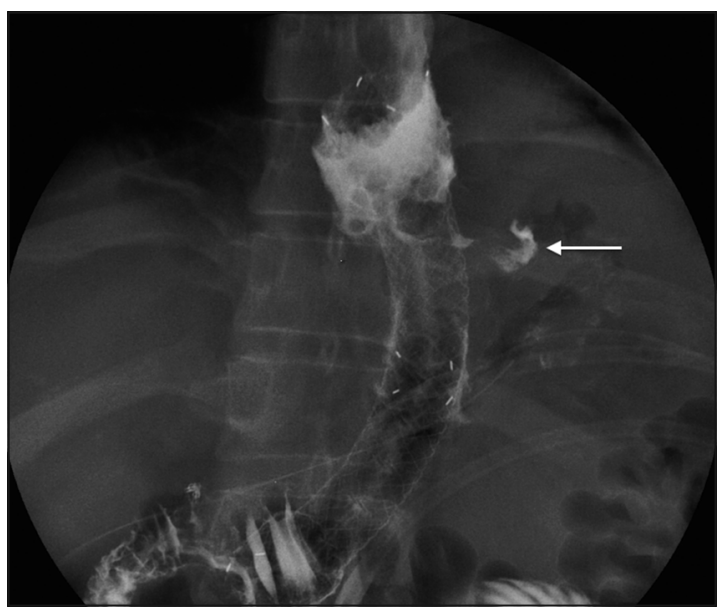

Figura 3. Tránsito esofagogastroduodenal con contraste hidrosoluble. Control de gastrectomía en manga complicada con filtración. La flecha indica material de contraste oral en la cavidad peritoneal; esto representa persistencia de una filtración, luego de la instalación de un stent endoscópico.

Hay que detallar los hallazgos, y diferenciar colección de gas, líquido y si presentan niveles hidroaéreos que pueden corresponder a un absceso.

Es posible drenar estas colecciones por vía percutánea bajo ultrasonido o TC por el radiólogo intervencionista ${ }^{5,6}$ (Figuras 5 y 6 ).

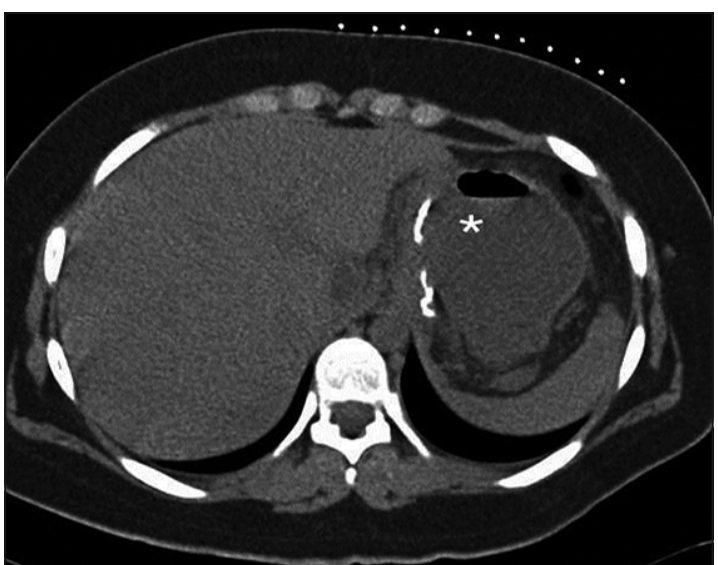

Figura 2. Tomografía computada de abdomen y pelvis no contrastada. Colección líquida con una burbuja de gas en su interior, adyacente a la línea de suturas de la manga, secundaria a filtración. Marcadores radio-opacos en el plano cutáneo para una eventual aspiración mediante radiología intervencional.

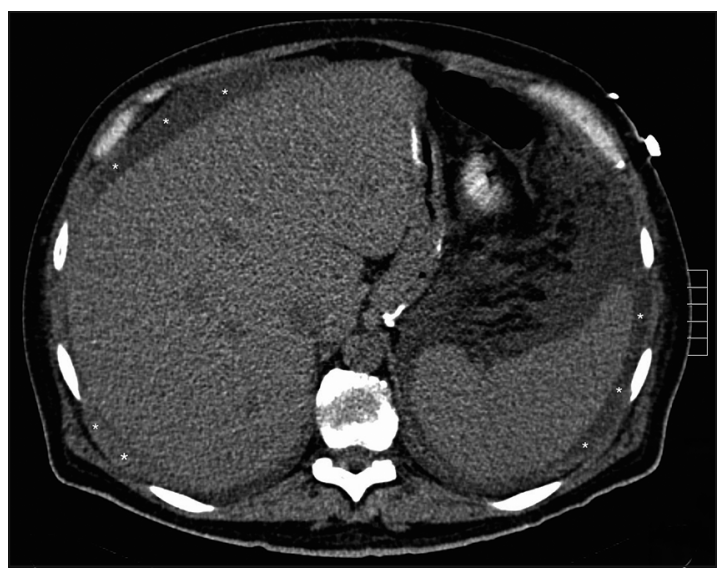

Figura 4. Tomografía computada de abdomen y pelvis no contrastada. Líquido libre adyacente al lecho quirúrgico e intraperitoneal, con componente espontáneamente denso en la región peri-hepática, los que representa hemoperitoneo $(*)$

\section{Fístulas}

Se originan a partir de filtraciones no tratadas, que con el tiempo pueden formar trayectos. Suelen encontrarse entre la pared gástrica y la piel, o entre la pared gástrica y otros segmentos del intestino o árbol bronquial ${ }^{2,5,7}$.

El tránsito esofagogastroduodenal puede mostrar paso de contraste oral a la piel, intestino, o menos frecuentemente al árbol tráqueo-bronquial. Puede ser necesario el estudio con TC para demostrar la 
comunicación y evaluar además posibles colecciones subfrénicas, mediastínicas o pleuro-pulmonares.

\section{Estenosis}

En los primeros días del post-operatorio es principalmente secundaria a edema o isquemia; la fibrosis, en tanto, suele ser la causa en estenosis tardías. Es más frecuente a nivel de la incisura angularis o en relación al sitio de alguna lesión gástrica previa cicatrizada ${ }^{9}$. El método de evaluación recomendado es el tránsito esofagogastroduodenal con contraste oral hidrosoluble, que muestra una limitación al paso de contraste a través del remanente gástrico. Dependiendo de la severidad y evolución de la estenosis,

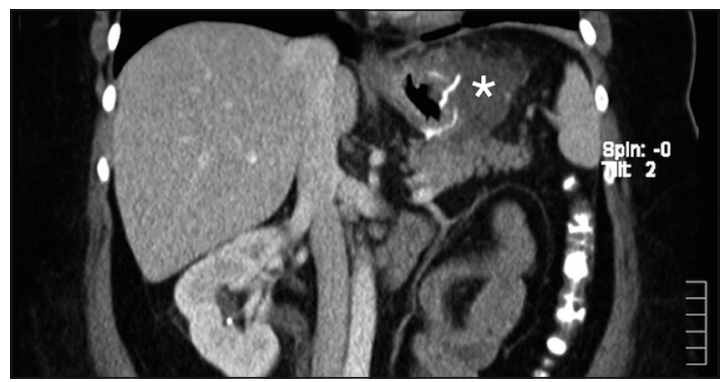

Figura 5. Tomografía computada de abdomen y pelvis con contraste en fase venosa; reconstrucción coronal. Colección líquida adyacente a la línea de sutura de la gastrectomía en manga $(*)$.

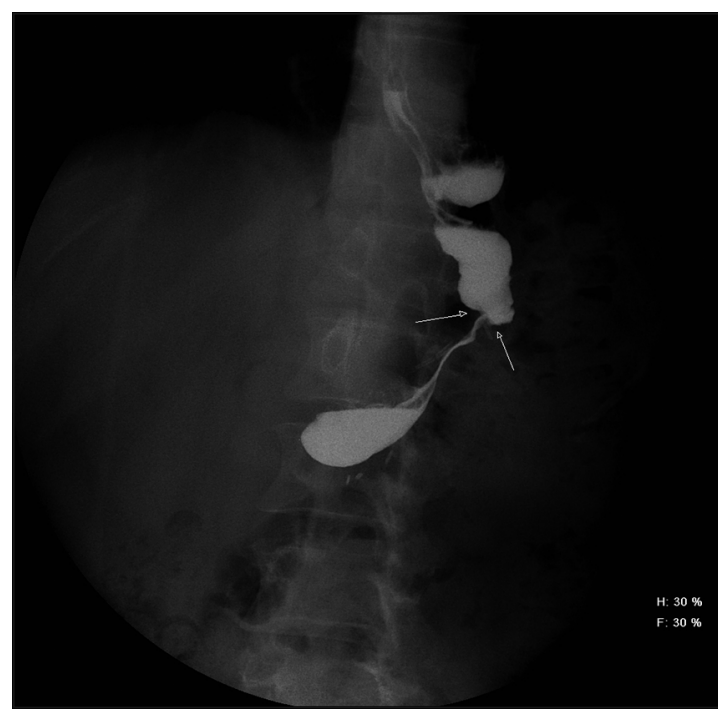

Figura 7. Tránsito esofagogastroduodenal con contraste hidrosoluble. Control de gastrectomía en manga reciente. Las flechas señalan una estenosis severa con flujo filiforme a distal. puede tratarse en forma conservadora con sonda gástrica e inhibidores de bomba de protones, o requerir tratamiento endoscópico o quirúrgico ${ }^{2,10}$ (Figura 7).

\section{Otras complicaciones}

Dentro de las complicaciones del período intraoperatorio se describe el desarrollo de infartos esplénicos, especialmente del polo posterior, debido a la ligadura de vasos cortos de la región fúndica ${ }^{11}$ (Figura 8).

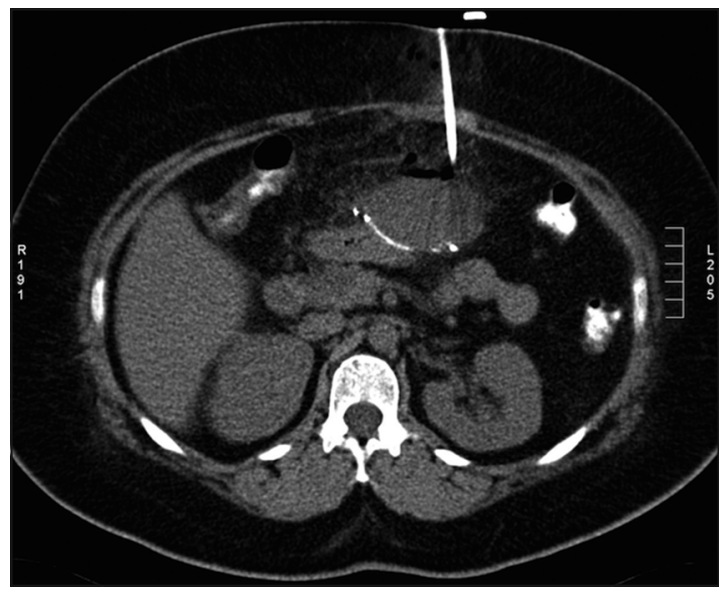

Figura 6. Tomografía computada de abdomen y pelvis no contrastada. Drenaje percutáneo de la colección previamente descrita, guiado por tomografía.

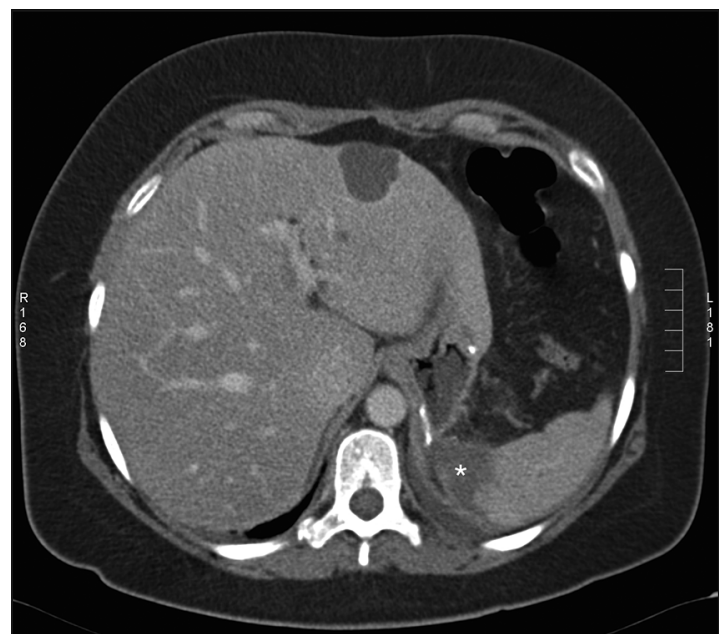

Figura 8. Tomografía computada de abdomen y pelvis con contraste en fase venosa, post gastrectomía en manga laparoscópica: área cuneiforme e hipodensa en el polo posterior del bazo $(*)$, lo que representa un infarto. Además se puede apreciar un quiste simple hepático intrascendente. 


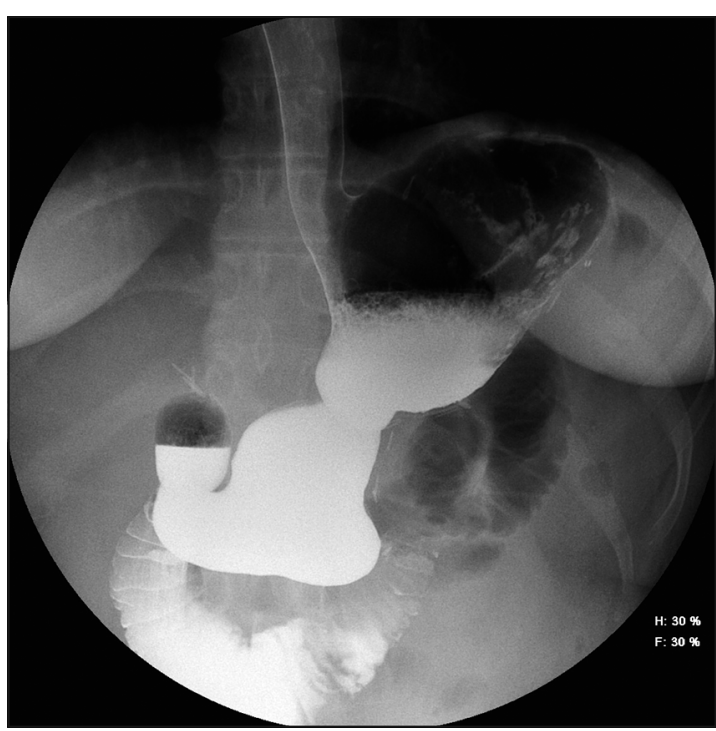

Figura 9. Tránsito esofagogastroduodenal con contraste hidrosoluble. Control de gastrectomía en manga: reflujo gastroesofágico severo, asociado a dilatación del remanente gástrico.

Como parte de las complicaciones tardías se describen déficits nutricionales (aunque menos severos que los producidos por el by-pass gástrico), caracterizados por déficit de vitamina B12, vitamina $\mathrm{D}$, calcio, folato, fierro y zinc. Por estos motivos se recomienda su monitorización mediante niveles séricos $^{12,13}$.

Otra de las complicaciones descritas es el desarrollo de enfermedad por reflujo gastroesofágico luego de la intervención. Se recomienda que sea tratado con inhibidores de la bomba de protones ${ }^{4,5,12,14}$ (Figura 9).

A mediano y largo plazo puede producirse aumento en el volumen del remanente gástrico si el paciente mantiene una gran ingesta, lo que suele asociarse a reganancia de peso $^{4,5}$ (Figura 10).

Además de las complicaciones previamente mencionadas, hay algunas menos frecuentes, poco descritas en la literatura, pero no por ello menos importantes. Una de ellas es el vólvulo del estómago post operado, que dado condiciones predisponentes como la laxitud en los ligamentos que fijan el estómago o una posición inusual del estómago luego de la cirugía, como consecuencia de la pérdida de estructuras que fijan la curvatura mayor, predisponen a la rotación de éste, que puede por lo tanto volvularse, tanto en el eje axial (vólvulo órgano axial), como en el eje del mesenterio (vólvulo meséntero axial). El vólvulo del intestino post operado ha sido reportado

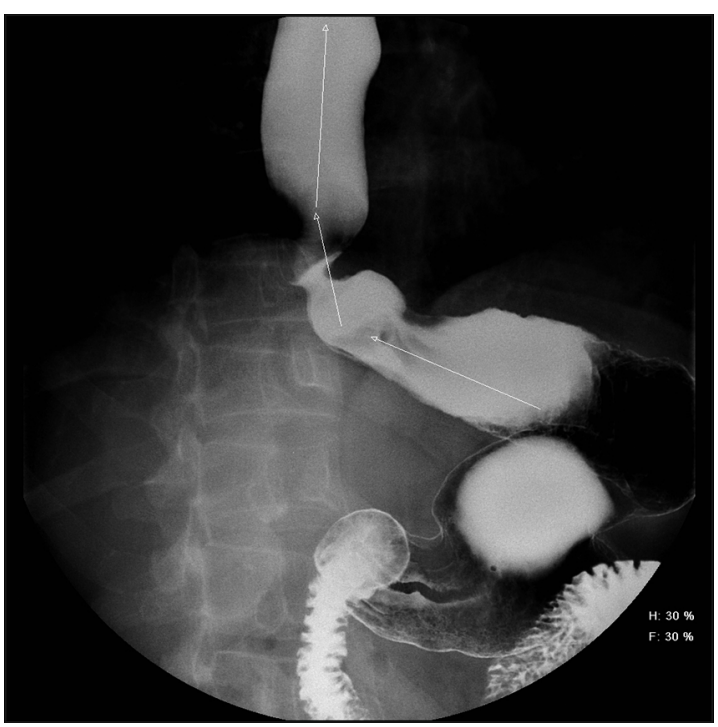

Figura 10. Tránsito esofagogastroduodenal con contraste hidrosoluble. Seguimiento a largo plazo de una gastrectomía en manga: dilatación del remanente gástrico mayor a la esperada, debido a sobre-ingesta alimentaria.

como una complicación precoz, caracterizado por un cuadro de obstrucción intestinal alta completa o parcial $^{15,16}$ (Figura 11).

Otra complicación infrecuente es la herniación intra-toráxica del estómago residual a través de un defecto diafragmático previo, la cual puede presentarse en el período post operatorio inmediato, o ser una complicación más tardía (Figura 12).

\section{Conclusión}

Las cirugías bariátricas son un tratamiento probado para la obesidad mórbida. Cada vez son realizadas con mayor frecuencia dado el alto índice de obesidad mórbida presente en la población.

El control de la gastrectomía en manga debe incluir un estudio fluoroscópico precoz de esófagoestómago-duodeno, donde pueda comprobarse un adecuado paso del medio contraste hidrosoluble a través del tubo gástrico. Este estudio también es útil para evaluar el peristaltismo gástrico e intestinal, además de mostrar complicaciones como filtraciones, estenosis o mal posiciones. La TC contrastada de abdomen y pelvis también es útil para evaluar filtraciones precoces, sin embargo, su rol principal es la detección de complicaciones como colecciones, sangrado, fístulas y/o hernias.

El conocimiento de las diferentes complicaciones 


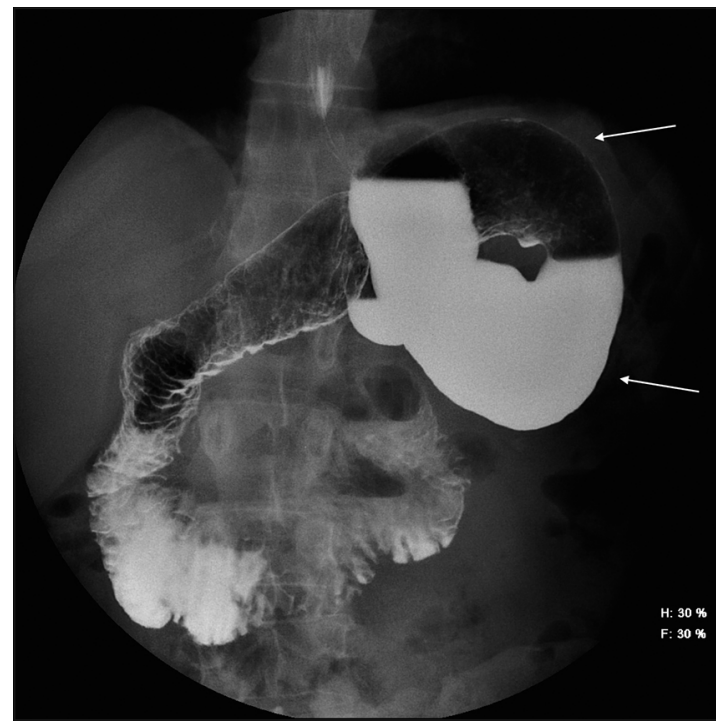

Figura 11. Tránsito esofagogastroduodenal con contraste hidrosoluble. Control de gastrectomía en manga: vólvulo meséntero-axial del remanente gástrico (la curvatura gástrica mayor tiene una ubicación craneal con respecto a la curvatura gástrica menor), en forma de "caracol".

de la gastrectomía en manga, tanto precoces como tardías, y el enfrentamiento mediante las técnicas de imágenes descritas permitirá un adecuado control postoperatorio y una disminución en la morbimortalidad frente a la presencia de complicaciones.

\section{Referencias}

1. OECD health statistics 2013. Panorama de Salud 2013 Informe OECD sobre Chile y comparación con países miembro. Http//dx.doi.org/10.1787/health-data-en.

2. Robert C. Chandler, Gujjarrapa Srinivas, Kedar N. Chintapalli. Imaging in Bariatric Surgery: A Guide to Postsurgical Anatomy and Common Complications. AJR 2008;190:122-35.

3. Guzmán S, Manrique M, Raddatz A. Results of bariatric surgery. Experience over 18 years. Rev Med Chile 2013;141:553-61. doi:10.4067/S003498872013000500001 .

4. Sucandy I, Antanavicius G, Bonanni F Jr. Outcome analysis of early laparoscopic sleeve gastrectomy experience. JSLS 2013;17:602-6.doi: 10.4293/108680813X 13693422520963.

5. Deitel M, Gagner M, Erickson AL, Crosby RD. Third International Summit: Current status of sleeve gastrectomy. Surg Obes Relat Dis. 2011;7:749-59.

6. Deitel M, Gagner M, Erickson AL, Crosby RD. The Second International Consensus Summit for Sleeve

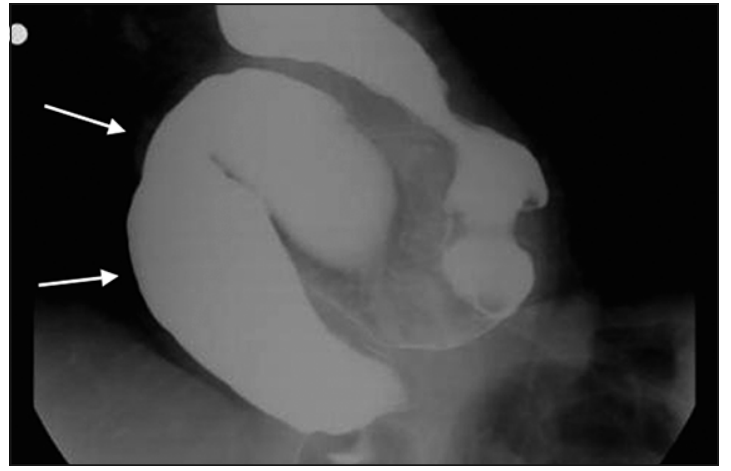

Figura 12. Tránsito esofagogastroduodenal con contraste hidrosoluble. Control de gastrectomía en manga: herniación intratorácica del estómago residual.

Gastrectomy. Surg Obes Relat Dis. 2009;5:476-85.

7. Moszkowicz D, Arienzo R, Khettab. Sleeve Gastrectomy Severe Complications: Is It Always a Reasonable Surgical Option? Obes Surg. 2013;23:676-86.

8. Alazmi W1, Al-Sabah S, Ali DA, Almazeedi S. Treating sleeve gastrectomy leak with endoscopic stenting: the kuwaiti experience and review of recent literatura. Surg Endosc. 2014;12:3425-8.

9. Sarkhosh K, Birch DW, Sharma A, Karmali S. Complications associated with laparoscopic sleeve gastrectomy for morbid obesity: a surgeon's guide. Can J Surg. 2013;56:347-52.

10. Chivot C, Robert B, Lafaye N, Fuks D, Dhahri A. Laparoscopic sleeve gastrectomy: Imaging of normal anatomic features and postoperative gastrointestinal complications. Diagn Interv Imaging. 2013;94:823-34.

11. Szewczyk T, Janczak P, Janiak A. Laparoscopic sleeve gastrectomy-7 years of own experience. Wideochir Inne Tech Malo Inwazyjne 2014;9:427-35.

12. Sarkhosh K, Birch DW, Sharma A, Karmali S. Complications associated with laparoscopic sleeve gastrectomy for morbid obesity: a surgeon's guide. Can J Surg. 2013;56:347-52.

13. Chiu S, Birch DW, Shi X, Sharma AM, Karmali S. Effect of sleeve gastrectomy on gastroesophageal reflux disease: a systematic review Surg Obes Relat Dis. 2011;7:510-5.

14. Carter PR, LeBlanc KA, Hausmann MG, Kleinpeter $\mathrm{KP}$, de Barros SN, Jones SM. Association between gastroesophageal reflux disease and laparoscopic sleeve gastrectomy. Surg Obes Relat Dis. 2011;7:569-72.

15. Del Castillo Déjardin D, Sabench Pereferrer F, Hernández González M. Gastric volvulus after sleeve gastrectomy for morbid obesity. Surgery 2013;153:431-3.

16. Paiva de Godoy, Eudes, Coelho, Daniel. Gastric sleeve fixation strategy in laparoscopic vertical sleeve gastrectomy. ABCD Arq Bras Cir Dig. 2013;26 (Suplemento 1):79-82. 